\title{
LA INTERROGACIÓN RETÓRICA A LA LUZ DE LA GRAMÁTICA DEL TEXTO
}

\author{
Joan G. Burguera Serra \\ Universidad de Barcelona \\ burguera@ub.edu
}

\begin{abstract}
Resumen
Las operaciones de cohesión y coherencia textual han sido analizadas, mayoritariamente, a partir del estudio de mecanismos léxicos o gramaticales. En este trabajo se propone una ampliación de las marcas de cohesión y coherencia prototípicas a partir de la descripción de la interrogación retórica como una estructura pragmática compleja encaminada a la articulación de funciones metadiscursivas de (i) apertura secuencial o textual, (ii) cierre secuencial o textual y (iii) avance discursivo. Asimismo, tomando en consideración que la interrogación retórica incorpora un componente de significado proposicional, se vincularán las funciones textuales señaladas con el desarrollo de diversas instrucciones argumentativas o con el asentamiento de una fuerza ilocutiva coorientada con el acto de habla global que se deduce del texto en el que se integra dicha estructura.
\end{abstract}

PALABRAS CLAVE: cohesión, coherencia, interrogación retórica, lingüística del texto, cartas al director

\begin{abstract}
Cohesion and coherence have been analyzed using lexical or grammatical mechanisms. In this paper, I propose a wide view of the archetypical items of cohesion and coherence. Thus, I expose a description of the rhetorical question as a complex pragmatic structure that operates not only as metadiscursive statement that is able to open or close sequences, but as a mechanism of the discourse advance. Equally, I pretend demonstrate that the textual functions of rhetorical questions are linked to the accession of an illocutive force, cooriented with the speech act of the whole text.
\end{abstract}

KEY WORDS: cohesion, coherence, rhetorical question, textual linguistics, editor letter

\section{Introducción}

Los estudios en torno al texto en tanto que unidad de análisis supraoracional con sentido comunicativo completo se han sustentado, por una parte, en la descripción de los mecanismos que permiten la cohesión de un conjunto de proposiciones articuladas de forma unitaria y, por otra, en el afianzamiento de la coherencia en relación tanto con los vínculos semánticos derivados de tales proposiciones, como con las variables pragmáticoenunciativas en las que se inserta la emisión del texto. En palabras de H. Calsamiglia y A. Tusón, la coherencia «es un concepto que se refiere al significado del texto en su totalidad, abarcando tanto las relaciones entre las palabras con el contexto como las relaciones entre las palabras en el interior del mismo texto», mientras que la cohesión «constituye una de las manifestaciones de 
la coherencia [...] que se da en el interior del texto y funciona como un conjunto de enlaces intratextuales para establecer las relaciones semánticas que precisa un texto para constituirse como unidad de significación» ${ }^{1}$.

En la descripción de los mecanismos de cohesión textual se han destacado cuestiones como el mantenimiento del referente mediante procedimientos léxicos o gramaticales, la conexión entre segmentos a través de marcadores, la existencia de diversas tipologías de progresión discursiva o de organización de los constituyentes, las correlaciones de tiempos verbales o las isotopías, entre otros. Sin embargo, más allá de estos mecanismos, raramente se ha abordado el análisis de otros procedimientos de cohesión llevados a cabo por estructuras sintáctico-pragmáticas complejas. Es, pues, el objetivo de este trabajo demostrar que la denominada interrogación retórica (en adelante IR) puede explicarse, desde una perspectiva eminentemente textual, como un elemento de cohesión y, por lo tanto, de coherencia, que opera tanto en el ámbito de la ordenación del texto como en lo relativo al avance discursivo.

Así pues, con el propósito de asentar esta hipótesis, en lo que viene procederemos a revisar sucintamente los principales mecanismos de cohesión analizados con recurrencia para, a partir de la caracterización de los denominados marcadores del discurso efectuada por A. Briz (1998), adaptar dicha propuesta descriptiva al estudio de las propiedades cohesivas de la IR. Ello debe posibilitar (i) la observación simultánea de las propiedades cohesivas y argumentativas de tal estructura desde una perspectiva textual y (ii) el afianzamiento de un parámetro discursivo explicativo que contribuya a sistematizar los cauces de incorporación de un significado procedimental de retoricidad en el seno de un enunciado interrogativo. Paralelamente, vincularemos este estudio a la aproximación secuencial que J.-M. Adam (1990, 1992, 2005) propone para abordar la diversidad de tipologías textuales. Ello implica, pues, partir de la idea de que un texto es un conjunto normalmente heterogéneo de secuencias, más o menos prototípicas, compuestas a su vez por grupos de macroproposiciones en cuyo seno se asientan las proposiciones individuales. Siguiendo a este autor, mantendremos que la ligazón textual deriva de la estructura composicional (la cohesión de una progresión lineal) y de la estructura configuracional (la coherencia), y que la IR contribuye a asentar estas dos grandes operaciones.

En nuestro caso en particular, la principal tipología secuencial que tomaremos como marco para explicar las propiedades textuales de la IR es la argumentativa. Esta elección viene determinada por el corpus de textos sobre el que se fundamenta este estudio: un conjunto de 150 cartas al director (en adelante CAD) de tres periódicos españoles de tirada nacional ( $A b c$, El País y La Vanguardia) en las que se observa la presencia de, al menos, un enunciado interrogativo cuya interpretación pragmática requiere una lectura retórica. En última instancia, se pretende mantener que la acomodación de la IR a la secuencia argumentativa debe explicarse en relación con uno de los macroactos de habla prototípicos de este género discursivo -la implementación de una queja o protesta- y en relación con los principales mecanismos de organización estructural del género epistolar de opinión.

Expuesto el marco metodológico de trabajo, propondremos una revisión de la noción de IR que supere su valoración como mera figura retórica y que, en consecuencia, permita un análisis de esta como estructura pragmática compleja. A partir de aquí, se desglosarán, por una parte, las funciones metadiscursivas de la IR, y nos centraremos con especial atención en

1 H. Calsamiglia y A. Tusón (1999), págs. 212, 220. 
las instrucciones de apertura y/o cierre de secuencia y en el uso retórico de la estructura pregunta-respuesta como mecanismo para la progresión textual. Simultáneamente, asociaremos a los usos metadiscursivos apuntados, una funcionalidad discursivopragmática relacionada bien con los constituyentes estructurales de los textos argumentativos, bien con la potencialidad pragmática del texto en el que se inserta dicha estructura. En último término, expondremos las conclusiones de este trabajo, poniendo especial interés en subrayar las perspectivas de análisis que, creemos, se deducen del estudio efectuado en torno a la IR.

\section{Mecanismos de cohesión textual y propuesta metodológica}

Los análisis de orientación textual, provengan bien desde la perspectiva de la Lingüística o la Gramática del texto ${ }^{2}$, bien desde una orientación más amplia como es el Análisis del Discurso, han tomado en consideración los procedimientos de cohesión del texto como un elemento capital en la descripción lingüístico-comunicativa de aquellas unidades que traspasan el ámbito oracional. Los estudios en torno a esta cuestión suponen un conjunto heterogéneo de aproximaciones a esta noción, no siempre coincidentes ${ }^{3}$. Sin embargo, más allá de las discrepancias existentes, entendemos en sentido amplio y de acuerdo con M. A. K. Halliday (1994: 309), que «cohesion can be defined as the set of resources for constructing relations in discourse wich transcend grammatical structure». Es decir, la cohesión implica, necesariamente, la complementación del estudio de los engarces de carácter léxico-gramatical mediante el estudio de los mecanismos que explican los vínculos en los que se sustenta el sentido unitario del texto; vínculos surgidos a su vez a partir de los significados semánticopragmáticos derivados del conjunto de estructuras proposicionales que conforman la totalidad del texto y que se generan, en consecuencia, sobre una base de coherencia. A modo de ejemplo, resulta difícil asumir que las relaciones deícticas endofóricas suponen un elemento de cohesión textual por el mero hecho de mantener un mismo referente en el desarrollo de la progresión textual. La correferencia debe explicarse, también, como un instrumento que contribuye a generar relaciones de significado transoracionales que constituyen, simultáneamente, la base de sentido para la correcta interpretación del texto en su globalidad.

Sea como fuere, pese a que la cohesión puede interpretarse como una condición necesaria para la aceptabilidad de un texto como tal, en ningún caso permite un análisis en el que sea valorada como una condición suficiente para la validez discursiva ${ }^{4}$. De hecho, muchos de los autores citados anteriormente mantienen que la cohesión es un aspecto del estudio de la coherencia. En este sentido, retomando a M. A. K. Halliday y R. Hasan (1976), podríamos afirmar que la cohesión otorga textura y que esta constituye, a su vez, un apartado de la coherencia.

Si avanzamos en la descripción de la cohesión como operación textual, veremos que en el estudio de los mecanismos que dotan de cohesión a un texto ha existido, a pesar de

\footnotetext{
2 Véase M. Casado Velarde (1993) para una distinción entre Lingüística del Texto y Gramática del Texto.

3 Véanse M.A.K. Halliday y R. Hassan (1976), E. A. Werlich, (1976), T. A. Van Dijk (1980, 1983), R. Beaugrande y W. U. Dressler (1981), G. Brown y G. Yule (1983), H. Mederos (1988), E. Bernárdez (1982, 1995) o J.-M. Adam (1990, 1992, 2005). E. Bernárdez (1995) esboza una relación de los múltiples significados que, según el tratamiento dado por diversos autores, se asocian a los términos cohesión y coherencia.

4 T. A. Van Dijk (1983: 84-85) «la conexión no depende de la presencia de conectivos [...] más bien el uso de los conectivos presupone que las oraciones están conectadas [...] Una primera condición de la conexión podría ser una relación entre los SIGNIFICADOS o SENTIDOS de las palabras de la frase».
} 
la multifuncionalidad del término, una cierta coincidencia a la hora de tratar determinados aspectos. Es más, en palabras de E. Bernárdez (1995: 129) «es posible analizar sistemáticamente los procedimientos de cohesión utilizados en una lengua e incluso aproximarse a sus rasgos universales, interlingüísticos». De ahí que el grueso estable de los estudios en torno a la cohesión textual en una gran diversidad de lenguas históricas se centre en los rasgos prosódicos, el mantenimiento léxico o gramatical del referente, la elipsis, los mecanismos de topicalización, la progresión temática, los marcadores o conectores discursivos, etc. En otras palabras, al igual que desde diversas perspectivas de análisis de la sintaxis oracional se han asentado principios de descripción universales, en el análisis textual, a los parámetros de estudio se les presupone un carácter supralingüístico puesto que pretenden ser de aplicación a cualquier texto independientemente de la lengua de configuración ${ }^{5}$.

A partir, pues, de la idea de universalidad cohesiva, esta propuesta descriptiva pretende adaptar los mecanismos de análisis aplicados a los marcadores del discurso (A. Briz, 1998), en tanto que categoría central de los procesos de cohesión, a la IR. En última instancia, proponemos un replanteamiento de la noción de cohesión con el fin de tomar en consideración la diversidad de tipologías textuales y géneros discursivos. Ello ha de facilitar la posibilidad de incorporar nuevos y específicos rasgos cohesivos asociados a cada una de estas. La idea que subyace a este análisis es que, conjuntamente con los procedimientos prototípicos, la cohesión puede ser tratada a partir de las idiosincrasias propias de cada tipología textual. Hablamos, si se quiere, de la complementación de una cohesión de alcance global mediante la introducción de parámetros de cohesión funcionales subordinados a los textos en relación con su macroestructura y su fuerza ilocutiva primaria. No se pretende, pues, equiparar la IR con los estándares de cohesión, puesto que, como hemos dicho, estos pueden entenderse como subyacentes a cualquier texto y la IR es un recurso de uso limitado. No se persigue tampoco asociar una funcionalidad cerrada a la IR en tanto que elemento textual. Nuestro objetivo reside fundamentalmente en dejar constancia de la posibilidad de vincular la IR con unas funciones metadiscursivas y pragmáticas altamente estables de gran relevancia para la cohesión y coherencia de los textos argumentativos.

Introducimos, pues, en lo que sigue una breve revisión de las dos principales aportaciones metodológicas que vamos a seguir a lo largo de este trabajo: A. Briz (1998) en lo relativo a los conectores pragmáticos como modelo descriptivo trasladable al análisis de la IR, y J.-M. Adam $(1992,2005)$ en lo referente a la estructura composicional y configuracional del texto.

El estudio de los marcadores del discurso que propone A. Briz (1998) en el marco del análisis de la conversación parte de una distinción inicial entre conectores pragmáticos argumentativos y metadiscursivos. Los primeros serían aquellos que se relacionan con las instrucciones de procesamiento de los enunciados que anexionan, mientras que los segundos administrarían la disposición estructural del texto o, dicho de otro modo, los primeros interfieren en la orientación y fuerza argumentativa, y los segundos en la distribución textual. Esta

5 J.-M. Adam (1992) mantiene que la competencia lingüística de los hablantes está supeditada a tres grandes «contraintes» (coacciones): las discursivas, que nos remiten a las prácticas discursivas histórica y socialmente consolidadas; las textuales, vinculadas a la heterogeneidad de la composición y organización de textos, y las locales o globales de una lengua específica que se relacionan a un plano fundamentalmente gramatical. El análisis de las marcas formales que inducen a consolidar una lectura retórica de un enunciado interrogativo se ubicarían en este último nivel, mientras que los usos y valores de la IR, en tanto que estructura pragmática, podrían asociarse tanto a las restricciones textuales como a las de género. 
clasificación binaria presenta, no obstante, unas fronteras difusas puesto que, según A. Briz, más que dos tipologías distintas de conectores, esta distinción pretende cubrir las diversas macrofunciones que pueden asociarse a un conector. Así, en un primer momento, se apunta que «el conector pragmático en español es conector de enunciados y conector de enunciadoenunciación; presenta un valor argumentativo y/o ilocutivo con unas consecuencias conversacionales determinadas» (Briz 1998: 177). Ahora bien, a continuación, al abordar el análisis de los conectores metadiscursivos se matiza que «más que de un conector distinto del conector argumentativo, se trata de la segunda función general de los conectores pragmáticos: la formulativa, la de resolver problemas de formulación, la de relacionar las partes articuladas con el todo» (Briz 1998: 204). El propio autor explica esta multifuncionalidad al afirmar que los conectores pragmáticos

constituyen una especie de agarraderos de habla, cuya función, más que en manifestar una relación argumentativa (sin por ello negar que en ocasiones la expresen o participen de ella) consiste en servir de apoyo a los interlocutores para formular y reformular sus mensajes y al mismo tiempo para agarrar y ordenar las partes de los mismos (Briz 1998: 199).

Con todo, de lo dicho no se deriva que no sea posible hablar de predominios funcionales, argumentativos o metadiscursivos, en ciertos conectores. El acento en una u otra función dependerá, en última instancia, de factores contextuales.

Esta propuesta descriptiva resulta aplicable a los valores de la IR en secuencias o textos argumentativos. En estos se puede apreciar que la IR desarrolla un papel metadiscursivo con tres variables de carácter formulativo: esta puede constituir un mecanismo de apertura de secuencia (o de la totalidad del texto), puede suponer el cierre de una secuencia (o de la totalidad del texto) o, en último término, puede funcionar como un mecanismo para la progresión discursiva. Ahora bien, paralelamente, la IR desarrolla dos funciones pragmáticas vinculadas con el enunciado y con la enunciación: así, la IR puede analizarse como un elemento consubstancial de la secuencia argumentativa que introduce la tesis, el garante argumentativo, la conclusión, etc. sin el cual se disipa la lógica discursiva de dicha secuencia y puede, paralelamente, actuar como anuncio o refuerzo del acto ilocutivo que se deriva de la totalidad del texto. El modelo de descripción de los marcadores propuesto por A. Briz (1998) refleja, pues, una adaptabilidad total en lo relativo al tratamiento de la IR aunque, evidentemente, existen claras diferencias entre las que destacamos los siguientes puntos:

a) La IR, a diferencia de los marcadores, dispone de una estructura sintáctica oracional no gramaticalizada. Es más, aunque necesariamente se integra en los parámetros constitutivos de la modalidad interrogativa, incorpora juntamente con el valor metadiscursivo o argumentativo, un contenido proposicional que no hallamos en los marcadores y que resulta relevante para la interpretación global del texto ${ }^{6}$.

b) La IR presenta, en tanto que estructura, unos valores cohesivos globales en lo que a la ordenación del texto se refiere, pero difícilmente pueden asociarse determinadas confi-

6 La atribución de un valor proposicional a la IR podría ser un argumento para analizar esta estructura como herramienta de coherencia textual. En todo caso, esta característica coincidiría con los procedimientos anafóricos o catafóricos que recuperan una información de carácter oracional y que, en consecuencia, disponen de un valor más allá del meramente cohesivo. 
guraciones gramaticales con el desarrollo de instrucciones concretas de, por ejemplo, apertura o cierre ${ }^{7}$. En el caso de los marcadores, bajo la categoría de marcadores organizadores del discurso, cada pieza o construcción se relaciona con una microfunción distributiva específica a la que, simultáneamente, puede añadirse un valor argumentativo o ilocutivo.

c) La cohesividad de la IR se vincula a ciertas tipologías textuales (la argumentación, la conversación) o a ciertos géneros discursivos (el discurso publicitario, el discurso político, etc.) mientras que los marcadores tienen un alcance lingüístico global.

Juntamente con la propuesta de A. Briz (1998), la disposición de este trabajo requiere enmarcar el análisis de la IR en una línea de análisis textual determinada. La propuesta de J.-M. Adam $(1992,2005)$ supone, en este sentido, una aproximación altamente interesante porque (i) toma en consideración la existencia de una fuerza ilocutiva como macroacto de discurso que, en nuestro caso, podremos asociar a la $\mathrm{IR}^{8}$; (ii) propone una descripción heterogénea de la estructura secuencial del texto que nos ayudará a explicar la imbricación de la IR en un género como el de las CAD y (iii) presenta un esquema secuencial del texto argumentativo (de lo prototípico a la periférico) que permite encajar el uso de la IR en relación con alguno de los constituyentes de la secuencia básica. De ahí que, antes de llevar a cabo el análisis pormenorizado de la IR como elemento cohesivo, sea conveniente revisar las bases de los esquemas de J.-M. Adam (1992, 2005) con el fin de establecer el marco en el que sustentar la explicación posterior. Este autor, a partir de los presupuestos de Bajtin, entiende que

un TEXTE peut être considéré comme une configuration réglée par divers modules ou sous-systèmes en constante interaction. Les trois premiers [la visée illocutorie, les repérages énonciatifs et la cohésion sémantique] correspondent à l'organisation qu'on peut dire pragmatique du discours; les deux derniers [la connexité textuelle et l'organisation séquentielle] permettent de rendre compte du fait qu'un texte est une suite non aléatoire de propositions (Adam 1992: 20).

A partir de esta definición inicial de texto, se incorpora una unidad de análisis intermedia entre la oración (proposición) y el texto entendido como producto final; la noción de esquema secuencial. Este concepto posibilita efectuar un acercamiento teórico para resolver algunas de las cuestiones relacionadas con la heterogeneidad y la jerarquización composicional que se observa en la mayoría de textos ${ }^{9}$. En este sentido, el concepto de secuencia debe entenderse como una entidad relativamente autónoma, dotada de organización interna y en relación de

7 Aunque partamos de la imposibilidad de vincular la estructura gramatical de la IR con un valor argumentativo o metadiscursivo concreto (apertura o cierre), cabe apuntar que sí se observan ciertas recurrencias que, tal vez, permitirían estudios futuros en torno a esta cuestión. Así, las IR introducidas por el pronombre indefinido alguien tienden a actuar como mecanismos de apertura secuencial, mientras que las IR articuladas bajo la forma general ¿Cómo es posible...? presentan, mayoritariamente, la incorporación de una fuerza ilocutiva que refuerza o justifica el acto global que se deriva de la totalidad del texto.

8 De acuerdo con D. Viehweger (1990: 49) "les analyses concrètes montrent que les actes illocutoires qui constituent un texte forment des hiérarchies illocutoires avec un acte illoctuif dominant étayé par des actes illocutoires subsidiarires".

9 J.-M. Adam mantiene (1992: 34) que «l'extrême hétérogénéité des genres de discours [...] est un constat empirique préalable à toute approche typologique des différences. L'hétérogénéité est une donnée que le linguistique ne peut pas ignorer». 
dependencia/independencia con el conjunto global del que forma parte ${ }^{10}$. De hecho, el propio autor propone el siguiente esquema (tabla 1) con el fin de evidenciar y sintetizar la jerarquía que subyace en el estudio del texto:

Tabla 1: Estructura textual jerarquizada (J. M. Adam, 1992)

[\# Texte \#[Séquence(s) [macro-propositions [proposition(s)]]]]

En el análisis concreto de la secuencia argumentativa, el autor esboza una primera distinción en relación con la noción de argumentación. Esta puede ser tratada bien desde una perspectiva del discurso y de la interacción social, bien desde la organización textual. Desde esta segunda vertiente, el estudio de la secuencia prototípica completa se resume de la siguiente manera (tabla 2):

Tabla 2: Esquema prototípico de la secuencia argumentativa (J. M. Adam, 2005)

Thèse antérieure + Données ------- Donc probablement ---------------- Conclusion
(nouvelle) these
Erincipes Base

Ahora bien, este esquema no responde ni a un orden lineal obligatorio ni a la presencia explícita de todos sus constituyentes. De hecho, siguiendo a este autor, «la (nouvelle) thèse peut être formulée d'entrée et reprise ou non par une conclusion qui la redouble en fin de séquence; la thèse antérieure et l'étayage peuvent être sous-entendus» (Adam 2005: 159). El aspecto más relevante de este esquema radica en que, de hecho, comporta dos niveles textuales distintos: el justificativo y el dialógico o contraargumentativo. En el primer caso, el destinatario adquiere un papel periférico, mientras que en el segundo la estructura argumentativa se supedita a un posible contraargumento derivado de un interlocutor real o potencial. Sea como fuere, esta explicación nos permite, en relación con el análisis que propondremos de la función argumentativa de la IR, dar cabida a dos cometidos distintos: (i) relacionar el contenido proposicional de la IR con cualesquiera de los elementos constituyentes de la secuencia argumentativa independientemente de su posición estructural y (ii) analizar el papel de la IR como elemento propiamente justificativo o como elemento dialógico. Expuestas las bases teóricas de este trabajo, corresponde ahora desarrollar las pautas cohesivas concretas que pretendemos atribuir a la IR.

10 No es el objetivo de este trabajo reproducir exhaustivamente la propuesta teórica de J.-M. Adam. Pese a que forman parte del acervo teórico de este trabajo, obviamos la exposición de conceptos de absoluta relevancia como son los de estructura secuencial dominante, combinación de secuencias, secuencia homogénea vs. heterogénea, plan de texto convencional vs. ocasional, etc. Todos ellos, no obstante, pueden consultarse en las obras señaladas (vid J.-M. Adam 1992, 2005). 


\section{La noción de interrogación retórica: definición y valores textuales}

La descripción de los valores cohesivos de la IR exige que previamente delimitemos qué entendemos por tal. Grosso modo, este fenómeno ha recibido dos tratamientos diferenciados a raíz de su valoración como figura estilística o como estrategia pragmática. Así, desde la teoría literaria, se ha sido descrito como una «figura literaria cuya finalidad no es indagar sino poner en evidencia y resaltar, con cierto énfasis y solemnidad, algo de lo que previamente se está seguro. Es un recurso expresivo que tiene por objeto provocar el asentimiento del oyente al mensaje que se le comunica» (Estébanez Calderón 1998: 570). Por su parte, los estudios lingüísticos se han centrado en aspectos tales como las funciones comunicativas que vehicula, la configuración gramatical de la estructura o los análisis derivados de un enfoque intercultural y/o traductológico ${ }^{11}$. Sin embargo, no podemos decir que se haya llegado a un acuerdo para afianzar una definición más o menos compartida. De hecho, de acuerdo con J. Frank (1990: 737), «the impreciseness in description and difference in approaches suggest that no one theoretical base is adequate to deal with the variability in form and function of rhetorical questions». Ahora bien, independientemente de la ausencia de una noción común de IR, sí se han señalado diversos parámetros que permiten efectuar una aproximación a este fenómeno. Entre ellos destacan el carácter netamente pragmático de la IR y, en consecuencia, su dependencia del contexto de emisión ${ }^{12}$ y la existencia de unas marcas formales que, pese a no ser determinantes, predisponen la lectura retórica de un enunciado interrogativo determinado. En palabras de J. Schmidt-Radefeldt,

rhetorical questions generally contain certain formal indications by which they are to be interpreted as «rhetorical»: Intonation pattern, special particles (adverbials), non-deontic modal verbs and verbal mood (conjunctive or conditional) are to be regarded as the main linguistic indicators for whether a sentence is to be regarded as rhetorical (assertive) or not. In case all these formal indicators are deleted in the surface structure of sentences (utterances) which are intented as rhetorical, the rhetorical speech act can fail insofar as it is interpreted as a genuine question (Schmidt-Radefeldt 1977: 381).

Otros aspectos como la exigencia o no de una respuesta, el asentamiento indirecto de una aserción con inversión de la polaridad del contenido proposicional de la estructura interrogativa o los papeles del emisor y el destinatario en el proceso de interpretación de la IR han generado, por el contrario, acercamientos divergentes.

Si nos centramos en el análisis de las funciones pragmáticas de la IR, deberemos señalar que uno de los principales objetivos que de forma general se le han atribuido radica en con-

11 Véanse, en este sentido, J. Schmidt-Radefeldt (1977), A. Borillo (1981), G. I. Anzilotti (1982), J. Frank (1990), C. Ilie (1994), C. Han (2002), I. Koshik (2005), H. Rohde (2006). En el ámbito hispánico debemos destacar los estudios de M. V. Escandell Vidal (1984, 1988), D. A. Igualada Belchí (1994), H. Haverkate (1994), J. González Calvo (1996), I. Carranza (2001), F. Fernández García (2002), entre otros.

12 C. Ilie (1994), desde una perspectiva marcadamente interaccional, destaca como parámetros de análisis a la hora de estudiar la IR, el tipo de discurso, la configuración contextual, la posición secuencial, la relación entre emisor y destinatario, los conocimientos y creencias compartidas, los roles y objetivos de ambos, la relación entre las intenciones del emisor y las expectativas del destinatario y el tipo de contestación (réplica o respuesta). 
firmar las creencias compartidas de los interlocutores sobre el mundo a partir, de acuerdo con H. Rohde (2006), del cumplimiento de tres condiciones de felicidad.

Their effect is to synchronize discourse participants commitments, confirming their shared beliefs about the world [...] I consider three felicity conditions that must hold between the answer sets of the Speaker and Addressee: (i) the presence of an obvious answer, (ii) the uninformativity of the answer, and (iii) the sufficient similarity of the Speaker and Addressee's answers (Rohde 2006: 135).

La obviedad de la respuesta, la no-informatividad de esta y la coincidencia entre la respuesta esperada por el emisor y la generada, explícita o implícitamente, por el destinatario se entienden como los parámetros definitorios mínimos para garantizar una simetría entre interlocutores. Ahora bien, más allá de las pautas de descripción, entendemos que el propósito global apuntado por H. Rohde (2006) enlaza directamente con lo que podríamos denominar función argumentativa del lenguaje puesto que persigue la implementación o la recuperación de los presupuestos del emisor en el destinatario. De hecho, sincronizar compromisos a través de la asunción de que los conocimientos sobre el mundo son compartidos por el emisor y por el destinatario, comporta que el primero parta de la base de que el destinatario asume ipso facto, o está dispuesto a incorporar como propio, el contenido implícito de la $\mathrm{IR}^{13}$. La persuasión o el acto argumentativo puede, en este sentido, valorarse como un proceso que debe consolidarse (el emisor piensa que el destinatario aceptará tácita y simultáneamente sus planteamientos) o como un mecanismo de refuerzo argumentativo (el emisor asume que el destinatario da validez de antemano a los presupuestos del primero). En el género discursivo que tomamos como punto de partida -las CAD- ambas situaciones son posibles, aunque predomina la segunda puesto que la situación comunicativa no se caracteriza ni por ser interactiva ni por ser inmediata ${ }^{14}$. Es más, a raíz de esta evidencia, adquiere relevancia el comentario de J. Frank al afirmar que «persuasive monologic contexts might be expected to yield higher frequencies of use of rhetorical questions» (Frank 1990: 727).

De lo comentado hasta el momento, se deriva que la mayoría de estudios pragmáticos en torno a la IR han focalizado el potencial suasorio o argumentativo de esta, pero no han contemplado, por ejemplo, el valor directivo que, en ocasiones, subyace a estas estructuras. Ello enlaza, en último término, con la valoración de la IR como una estructura con fuerza ilocutiva. Esto es, cuando utilizamos la IR como pieza de una secuencia argumentativa, esta estructura se reviste de un contenido proposicional determinado que contribuye a generar una lógica discursiva concreta. Sin embargo, en ocasiones y siempre tomando en consideración el contexto de emisión, la IR puede actuar como un elemento de refuerzo ilocutivo en relación con la finalidad comunicativa del texto en su globalidad. Este objetivo, juntamente con lo apuntado anteriormente, nos permite establecer tres niveles de análisis para la IR: el formal,

13 Este planteamiento implica que la noción de «espacio común» puede entenderse como condición necesaria en el emisor, pero no como condición suficiente para hablar, efectivamente, de una IR.

14 D. A. Igualada Belchí (1994) mantiene que la IR es frecuente en aquellos marcos comunicativos asimétricos donde se estable un diálogo ficticio: arengas políticas, homilías, cartas al director, etc. En estos casos, el emisor puede utilizar intencionalmente todos los medios a su alcance para comunicar mientras que el destinatario está privado, por la naturaleza misma de la situación, del uso de la palabra. Estaríamos ante lo que podríamos denominar un uso «falaz» de la IR. 
el textual y el pragmáticodiscursivo. En el primero, se tratarían aquellos elementos propiamente lingüísticos o gramaticales que actúan en la generación de un significado de retoricidad; en el segundo, se abordarían las funciones de la IR en relación con la organización de la secuencia textual en la que se integra y, en último término, se analizarían aquellos aspectos que adquieren relevancia a partir de la situación comunicativa concreta de emisión. En este trabajo, analizamos las relaciones existentes entre los dos últimos estadios.

\section{Valores argumentativos y metadiscursivos de la interrogación retórica en la organización de las secuencias textuales}

En los apartados anteriores ya hemos avanzado grosso modo qué funciones discursivas pueden asociarse a la IR. En tanto que elemento de cohesión, la IR supone mayoritariamente o bien un mecanismo de organización textual, como herramienta de apertura o de cierre secuencial y/o textual, o bien un medio de progresión discursiva y/o cambio de tópico. Paralelamente, desde una perspectiva argumentativa, la IR puede ocupar el papel de cualquiera de los elementos integrantes de la secuencia argumentativa prototípica descrita por J.-M. Adam $(1992,2005)$ (tesis, conclusión, garante, etc.) o actuar como un elemento de refuerzo ilocutivo relacionado con el acto de habla global en el que se inserta la IR en cuestión. En este sentido, y en relación con la propuesta metodológica apuntada en torno a la coincidencia de parámetros descriptivos entre la IR y los marcadores, aquella podría relacionarse con los marcadores del discurso distributivos (ordenadores, aditivos o reformuladores) en la medida en que ambas categorías contribuyen a perfilar una segmentación del texto en grupos de (macro) proposiciones con sentido unitario. Asimismo, al trasladar un significado proposicional indirecto, la IR desarrolla una función argumentativa similar a la que puede atribuirse a ciertos marcadores argumentativos. Ahora bien, mientras que los marcadores focalizan las instrucciones de procesamiento o interpretación de los elementos que anexionan, la IR constituye en sí misma un bloque de la cadena argumentativa. En lo que viene intentaremos, pues, justificar estos valores a la luz de los usos de la IR atestiguados en el corpus de trabajo.

El uso metadiscursivo más frecuente que cabe atribuir a la IR se relaciona con la función de cierre secuencial y/o textual. En estos casos, la IR actúa, en términos de organización textual, a modo de marcador de conclusión o cierre. Es decir, la IR supone, pese a constituir una construcción abierta desde la perspectiva de la prosecución discursiva y como consecuencia de su formato interrogativo, la última proposición con la que el emisor decide finalizar bien una secuencia textual, bien la totalidad del texto. Tal disposición podría explicarse a raíz de la voluntad del emisor de abrir un pseudoespacio de interacción en el que el binomio enunciado-pregunta actúe como una estructura inconclusa que posibilita el final comunicativo mediante la réplica (explícita o mental, inmediata o mediata) de un hipotético destinatario. Sin embargo, en este punto hay que recordar que la IR no se caracteriza por buscar una respuesta informativa, en el sentido estricto del término, puesto que no forma parte de su cometido pragmático articular un acto de habla de pregunta. Busca obtener la confirmación. Es, de hecho, el asentamiento de un acto de habla indirecto de carácter mayoritariamente asertivo lo que posibilita el uso de esta estructura en posición final. Es más, en ocasiones es plausible hallar la presencia de un comentario posterior que no se interpreta como la respuesta a la supuesta pregunta planteada, sino como una anotación última construida sobre 
la inferencia derivada de la interpretación retórica del enunciado interrogativo previo, tal y como se observa en (1).

\section{(1) También soy español}

Como empresario catalán y español, jamás pensé que estar en mi tierra iba a ser cultivo de tales fascismos por parte de los políticos de la Generalitat. Es indignante que se haga una persecución de todo lo que sabe a español (imposición del catalán). ¿No recuerdan esos mismos políticos que esa actitud la tuvieron en la dictadura contra la lengua catalana, y que en Cataluña eso siempre nos ha dolido? Sólo van a conseguir que empresas como la mía, y muchas otras, abandonen las tierras catalanas.

Soy catalán y siempre lo seré, pero respeten también mi condición de español. Siempre hemos sido felices como estábamos, ¿Quiénes son ustedes para implicarse en mi sentimiento de ser español?, ¿quiénes son ustedes para que el resto del Estado nos mire como insolidarios y separatistas?, ¿quiénes son ustedes para que nos juzguen a todos por su incapacidad para gobernar? Señor Zapatero, gracias por crear el peor clima que jamás ha vivido este país.

Jesús Martín [ABC, 23/01/2006]

En este caso, el segmento Señor Zapatero, gracias por crear el peor clima que jamás ha vivido este país no se analiza como la respuesta a la retahíla de interrogaciones previas, sino como la explicitación asertiva de un acto de habla de queja, de carácter marcadamente irónico, que adquiere el valor de conclusión final tras el conjunto de reproches argumentativamente coorientados que subyace a la serie de IIRR.

El uso metadiscursivo de final o cierre de la IR permite, asimismo, establecer ocasionalmente un criterio discursivo para identificar el valor pragmático de retoricidad de un enunciado interrogativo. En otras palabras, la retoricidad puede hallar una explicación en el marco de la deixis textual a través de mecanismos anafóricos. El discurso previo puede aportar, explícita o inferencialmente, una información que a la postre suponga la respuesta a la pseudopregunta planteada mediante la estructura interrogativa. Ello invalida, pues, la posibilidad de interpretar tal enunciado como una auténtica pregunta puesto que previamente el propio emisor ha aportado lo que sería la respuesta a la misma y, en consecuencia, no debe resolverse ninguna incógnita. En este caso, la IR puede analizarse como una estrategia de reformulación discursiva en tanto que retoma un contenido previo que replantea mediante una modificación formal (de la aserción directa a la aserción indirecta o, si se quiere, de la aserción a la interrogación), y como un elemento de refuerzo argumentativo por el hecho de incidir en un contenido ya dado y, por lo tanto, ya conocido. En todo caso, resulta evidente que la IR actúa como un elemento cohesionador ya sea por el mantenimiento de una referencialidad determinada, ya sea por la pautas de distribución textual que lleva asociadas. Observemos, en todo caso, otros ejemplos.

(2) El congreso del PP

Congreso o convención, qué importa. Lo que conviene es reunirse un grupo numeroso de amigos bajo las mismas siglas de partido, contarse lo estupendos que son y atraer a la prensa para aparecer gratis en las portadas de los periódicos y de los telediarios. Ríen, hablan de política, critican al rival y deciden sobre el papel el futuro del país en el que cuentan con gobernar. Es ridículo que el PP hable de renovar el partido. ¿Cómo va a renovarse si ha derivado de un falso centrismo a una derecha descarada sin cambiar 
a sus dirigentes (Rajoy, Aznar, Zaplana, Acebes) bajo la inspiración en la cúpula de un ex ministro franquista?

Queda bien para el consumo de los adeptos la fotografía de Rajoy y Aznar sentados en el suelo, sonrientes y felices, en plan juvenil, cuando en realidad son unos carcas. Una vez más, Aznar no ha desaprovechado la ocasión para mentir y asegurar que no pactó con ETA, cuando hizo lo que Zapatero no se ha atrevido a hacer: acercar a más de cien terroristas encarcelados al País Vasco. Pero es que Aznar no renuncia a ser el Pinocho de la política española.

Antonio Nadal [La Vanguardia, 06/03/2006]

En (2) la IR supone el cierre final de una secuencia argumentativa intratextual. En este caso, la secuencia textual se organiza mediante una disposición u orden regresivo en el que se incorpora, en primer lugar, la conclusión o nueva tesis y, a continuación, las premisas o argumentos sobre los que se sustenta. En palabras de J.-M. Adam (1992: 115) «l'ordre régressif est plutôt celui de la preuve et de l'explication». En este caso, la oración Es ridículo que el PP hable de renovar el partido actúa simultáneamente como el elemento $\mathrm{P}$ en el esquema explicativo-causal [ $\mathrm{P}$ - ya que - $\mathrm{q}]$ y como la información discursiva previa que predispone la lectura retórica del enunciado interrogativo posterior, a la postre el elemento q. Es decir, anafóricamente se ha ofrecido la respuesta a la supuesta pregunta planteada, luego la interpretación retórica es la única posible para la lectura lógico-discursiva del texto. De hecho, el enunciado interrogativo no plantea verdaderamente una incógnita de carácter modal puesto que el pronombre interrogativo cómo no busca descifrar realmente de qué manera va a renovarse el PP. La IR incorpora un conjunto de informaciones (El PP ha derivado de un falso centrismo a una derecha descarada o El PP no ha cambiado a sus dirigentes, entre otras) que sirven de justificación para la aserción anterior que, como se ha apuntado, se reintroduce en la progresión discursiva mediante la interpretación inferencial con cambio de polaridad de todo el enunciado interrogativo. El procedimiento de interpretación a través de la anáfora textual y la duplicación referencial -se insiste doblemente, explícita e inferencialmente, en la imposibilidad de hablar de renovación del PP-contribuyen, pues, a generar cohesión y, en consecuencia, coherencia textual.

Hasta el momento se ha podido observar tanto en (1) como en (2) la combinación en la IR de una función metadiscursiva de cierre (textual y secuencial) conjuntamente con el desarrollo de diversos valores argumentativos estructurales (garantías o premisas de paso). Sin embargo, en la exposición de las funciones argumentativas que vinculamos a la IR se ha señalado la posibilidad de que esta desarrolle una función pragmática de orientación y/o refuerzo ilocutivo. En estos casos, la IR no asume un papel estructural en la organización de una secuencia argumentativa determinada ${ }^{15}$, sino que funciona como un mecanismo que canaliza la actitud del emisor ante el contenido del texto o, en otras palabras, especifica en qué acto de habla se inserta el texto. De algún modo, la IR sintetiza la justificación o motivo que induce al emisor a la composición de la carta en cuestión. En este sentido, la IR, pese a mantener un contenido proposicional concreto, enlaza directamente con la enunciación.

15 Obsérvese que en (1) y (2) la elisión de las IIRR exigiría una reconfiguración del material discursivo. Sin embargo, en (3) la eliminación de la IR no implica necesariamente un replanteamiento de la disposición textual presentada, dado que la IR no desarrolla un papel consustancial en esta secuencia argumentativa. 
(5) La voz de los profesores

Como ciudadanos, como padres de alumnos de secundaria y como profesionales de la enseñanza, ya que ambos somos profesores de instituto, queremos expresar nuestro descontento y nuestra preocupación ante la aprobación de la LOE por el Gobierno actual. En nuestra opinión, esta ley contiene múltiples deficiencias, pero vamos a resaltar solamente dos de ellas, que ya por sí mismas serían suficientes para que, haciendo uso del sentido común y del sentido de la justicia, esta ley jamás debería haber sido aprobada. La primera y la más grave es el hecho de que con dicha ley no se garantiza a los padres los derechos y libertades que en materia educativa reconoce la Constitución (libre elección de centros, elección del tipo de educación que se prefiere para los hijos...). La segunda es que, con dicha ley, no se resuelve el alto índice de fracaso escolar, pues se reiteran algunos errores de la LOGSE, no se fomenta la cultura del esfuerzo, ni se introducen medidas que apoyen y prestigien la autoridad y trabajo de los profesores.

¿Cómo es posible que no se escuche la voz de quienes de verdad defendemos la libertad y la calidad de la enseñanza?

José Gerardo Conde y Olga $\mathrm{M}^{\mathrm{a}}$ Suárez [ABC, 17/01/2006]

En (3) hallamos una secuencia argumentativa que se compone de los siguientes elementos: la preocupación ante la aprobación de la LOE por el Gobierno actual (tesis inicial), la falta de libertad de elección de los padres en materia educativa (premisa), la reiteración en algunos errores de la LOGSE (premisa) y la inutilidad que se deriva de la aprobación de esta ley (conclusión). La IR incorpora un acto de habla indirecto de carácter directivo que puede reconstruirse de la siguiente manera: debe escucharse la voz de quienes de verdad defienden la libertad y la calidad de la enseñanza ${ }^{16}$. Ciertamente, por su contenido proposicional inherente, podría argüirse que la IR es propiamente argumentativa porque, de hecho, incorpora una nueva premisa que apunta en la misma dirección que las dos anteriores. Sin embargo, el contenido de la IR no se presenta como una de las «deficiencias» que subyacen, según los autores, a la LOE. La IR focaliza, en este sentido, el acto de habla de queja o protesta de los emisores ante los hechos descritos. Supone, como comentábamos, la justificación de la emisión de todo el texto. Funciona, de hecho, como un operador ilocutivo en tanto en cuanto permite resumir, y de ahí su posición final, la fuerza ilocutiva que se halla tras esta CAD. De ahí que la posición final adquiera una lógica discursiva evidente porque supone un trasvase del plano enunciativo al plano de la enunciación. En (3) la IR -insistimos-, pese a disponer de un material potencialmente argumentativo, se sitúa en el ámbito de la emisión para reforzar la tipología de acto de habla en el que se inscribe la texto.

Paralelamente a la función vinculada con el cierre textual y/o secuencial, claramente mayoritaria, la IR asume en ocasiones una posición inicial y, por tanto, un valor metadiscursivo de apertura textual y/o secuencial. En estos casos, a diferencia de los comentados hasta el momento, la retoricidad no puede venir determinada anafóricamente por la información

16 Pese a que mayoritariamente la IR implica la existencia de una aserción indirecta, en ocasiones, como consecuencia de un doble proceso de interpretación inferencial, de la aseveración indirecta primaria se deriva, posteriormente, un acto directivo en el que se transmite una contenido proposicional bajo una modalidad deóntica de obligación. 
aportada en el discurso previo ya que esta no existe, es decir, el valor retórico de la estructura interrogativa emana de la existencia de un conocimiento o premisa que el emisor entiende como consabido o tópico, tal y como sucede en (4) y $(5)^{17}$. El procedimiento para explicar la retoricidad no es ya de índole textual, sino que se relaciona directamente con las pautas que configuran la situación comunicativa (la relación entre el emisor y el destinatario, la finalidad comunicativa del primero, el grado de conocimiento compartido, etc.) y, en el caso concreto de las CAD, el valor retórico surge en la mayoría de ocasiones como consecuencia de fundamentar el enunciado interrogativo sobre un topos cultural.

\section{(4) Justicia preventiva}

¿Alguien se puede imaginar que le obliguen a pagar un impuesto especial cuando se compra un simple cuchillo de cocina porque se puede usar para causar daños a otras personas? ¿O incrementar el precio de los coches porque pudieran estar implicados en un atraco mediante el método del alunizaje? ¿Y si además este incremento se abona a una empresa y no al Estado?

Situaciones como éstas que pueden parecer absurdas se están dando en el ámbito de los soportes informáticos con total impunidad y con el beneplácito de las leyes. Se puede aceptar que los socios de la SGAE cobren cuando se utilizan sus obras, pero es inconcebible que tengamos que pagar cuando no los usamos. Como informático, utilizo los discos para almacenar datos que nada tienen que ver con esta empresa, y sin embargo debo pagar el canon por cada uno de ellos. Es deplorable la actitud de la SGAE, pero más aún lo es de los legisladores que la permiten. No se les vaya a ocurrir extrapolarlo al Código Penal y tengamos que pasar todos un año en la cárcel por si acaso cometes algún delito en el futuro.

Pablo Urcola [El País, 10/03/2006]

En (4) se nos presenta una reflexión crítica en torno a los impuestos que gravan determinado material informático. El hecho de proceder al inicio del desarrollo discursivo mediante lo que podríamos denominar una «estructura interrogativa múltiple de uso retórico» se justifica, desde una perspectiva argumentativa, como consecuencia de la voluntad del emisor de asentar un argumento que, a través de un procedimiento de analogía, aporte un elemento o premisa que pueda ser objeto de comparación en relación con el discurso posterior. Desde la perspectiva de la organización textual, el valor retórico resulta indispensable para acotar un cierto grado de cohesión y, por lo tanto, de coherencia textual. De hecho, si los enunciados interrogativos permitieran una interpretación en tanto que preguntas, a las que pudieran seguir respuestas del tipo mucha gente imagina ... o todo el mundo imagina..., el segundo párrafo perdería lógica o coherencia discursiva, puesto que el propio emisor califica las situaciones apuntadas como «absurdas». Se produciría, pues, una contraargumentación en el seno del mismo discurso ya que, por una parte, podrían aceptarse como válidas las prosecuciones arriba propuestas y, paralelamente, describirlas como un sinsentido. La orientación argumentativa del texto objeto de análisis parte de la interpretación retórica con cambio de polaridad

17 Esto es así, sobre todo, en los casos en los que la IR implica el inicio o apertura del texto. En el caso de hallarnos ante aperturas de secuencias intratextuales de carácter básicamente argumentativo, la IR puede remitirnos a información previa ya conocida que, en todo caso, no suele ser determinante para el asentamiento del significado procedimental de retoricidad. 
de la estructura interrogativa inicial (nadie imagina que...) y de la consiguiente valoración negativa de tales estados de cosas. Es, pues, evidente que en estos casos la retoricidad no viene determinada por una material discursivo previo, sino por la suma de un topos y de una prosecución temática concreta que, a la postre, invalida la posibilidad de procesar los enunciados interrogativos como verdaderas preguntas. Luego, la retoricidad cohesiona, puesto que de ella depende la lógica organización de la información o material discursivo.

(5) El Palacio Real abre su galería de pintura

1 de marzo. Acabo de abrir la edición de su periódico tratando de acertar con la sección de Deportes; no lo consigo y acabo en cartelera, y leo «El Palacio Real abre su galería de pintura». Hombre, qué suerte, yo trabajo justo al lado, puedo aprovechar la hora de la comida y verla: Juan de Flandes, Caravaggio, Velázquez, Goya, qué bien. Continúo la reseña hasta el final: entrada, 10 euros, y joh! desilusión.

¿Alguien piensa que cobrando 1.000 euros escasos al mes puedo permitirme gastar 10 en esto? Desde luego, si lo piensa es porque no tiene ni idea de lo que es vivir con esa cantidad. Lamentablemente, muchas cosas no han cambiado ni parece que vayan a hacerlo; la cultura, en general, y el arte, en particular, siguen sin estar al alcance de todos en este país. ¿Será porque nuestros dirigentes piensan que la sensibilidad artística y cultural del individuo va en consonancia con su nivel económico? La respuesta es no por ahora, pero visto el cariz que han tomado las políticas educativas y culturales en los últimos tiempos, todo se andará.

César Galiano [El País, 04/03/2006]

En (5), por su parte, hallamos un texto heterogéneo en el que se entremezcla una secuencia narrativa y otra argumentativa, esta última dominante desde la perspectiva jerárquica de relevancia comunicativa. En esta muestra, la IR actúa como mecanismo de apertura secuencial que implica, simultáneamente, el cambio de tipología secuencial de la narración a la argumentación. La IR permite, pues, que este trasvase de secuencias se lleve a cabo sin quebrantar el devenir discursivo que pretende el emisor. Es más, la IR se reviste de una función valorativa en torno a la situación descrita en la secuencia previa, puesto que traslada la siguiente idea: Todo el mundo sabe que cobrando 1000 euros NO puedo permitirme gastar 10 en esto en la que el pronombre esto nos remite a la situación anteriormente descrita. De nuevo, la interpretación del enunciado interrogativo como pregunta tendría un encaje anómalo tanto con el discurso previo -obsérvese la evaluación de la descripción mediante la expresión joh! desilusión - como con el proceder discursivo posterior en el que se explicita la imposibilidad de dar una hipotética respuesta afirmativa a la supuesta cuestión planteada. Desde el análisis del cometido argumentativo, la IR de (5) permite una explicación en la que se dé cabida tanto al asentamiento de un elemento estructural en el seno de la secuencia argumentativa (una premisa que apoya la ulterior conclusión la cultura y el arte no están al alcance de todos), como a la implementación de un acto de habla, relacionado nuevamente con la enunciación, mediante el cual el emisor presenta el potencial crítico que se deriva de la totalidad de la CAD. Es más, en contraste con lo que sucede en (3), en (5) la IR anticipa la funcionalidad del texto. Es decir, en (3) la IR refuerza la denuncia como macrofinalidad textual y de ahí su posición final. Por su parte, en (5) la IR preestablece, más allá de su uso como premisa o hecho argumentativo, una macrofunción discursiva y de ahí, en consecuencia, su posición inicial. 
Siguiendo con el análisis propuesto, hay que detenerse, en último término, en aquellos casos en los que la IR supone un elemento de progresión discursiva intratextual, tanto en el marco de estructuras bipolares como en el marcaje explícito del par de adyacencia pregunta-respuesta ${ }^{18}$. En el primer caso, la interpretación retórica de un determinado enunciado interrogativo viene exigida por la cohesión propia de aquellas estructuras oracionales bipolares en las que un segmento determinado no se presenta de forma explícita, sino inferencial. Por su parte, el uso retórico del binomio pregunta-respuesta responde a una estrategia discursiva en la que el bloque pregunta actúa como mero material metadiscursivo para la incorporación textual del fragmento asertivo respuesta. Ahora bien, en estos casos, la implementación de la respuesta viene dada por la voluntad del emisor de reafirmar aquel contenido discursivo que, inferencialmente, ya se ha proporcionado. En otras palabras, el uso retórico del par pregunta-respuesta se fundamenta en la primaria interpretación retórica del enunciado interrogativo independientemente de la presentación posterior de una aserción que desarrolle el papel de respuesta. Per se, la estructura interrogativa orienta hacia una aserción indirecta que, finalmente, se manifiesta lingüísticamente mediante la incorporación de material textual que dota de forma lingüística al significado previamente adquirido. En ambos casos, pese a disponer de un potencial argumentativo relevante para el afianzamiento de una determinada secuencia, es prioritaria la función metadiscursiva de la IR, puesto que contribuye a reconstruir una estructura incompleta (véase, por ejemplo, el caso de las condicionales) o a reduplicar (o reformular) un contenido determinado.

(6) Ayer y hoy

Declararon e hicieron la guerra prescindiendo de la ONU, en un alarde de arrogancia. ¿Para qué la necesitaban? Pero eso fue ayer. Hoy reclaman su ayuda para reconstruir lo que ellos destruyeron, que ironía. Para justificar su decisión argumentaron como finalidad la captura del dictador, la eliminación de armas de destrucción masiva y la seguridad del mundo. Hoy Sadam está libre, no hay rastro de las armas y el mundo dista mucho de ser un lugar más seguro que ayer. Pero la guerra se llevó a cabo y finalizó, eso nos dijeron, aunque no sabemos si creerlo, porque hoy nos dicen que desde entonces han muerto más soldados que los que murieron antes. Ayer a la ocupación la llamaron liberación y hoy a la resistencia la llaman terrorismo. Ayer Irak era una abominable dictadura, es cierto, pero hoy es un país semidestruido e inmerso en el caos, donde lo único que funciona son unos cuantos pozos de petróleo. ¿Mereció la pena? ¿De verdad es mejor la situación de hoy que la de ayer?

Sebastián Fernández [El País, 01/10/2003]

(7) Personas normales

Es incompresible -mejor dicho, patético- que en pleno siglo XXI siga habiendo gente que cambie su forma de tratarte al enterarse de que eres homosexual. Yo estoy viviendo esta situación. Por suerte, sólo me ha pasado con conocidos, no con amigos. Pero aun así, me duele pensar que haya gente que te trate de modo distinto, casi despectivo, al

18 Es importante remarcar que J.-M. Adam (2005) señala que el esquema [problema (pregunta) - explicación (respuesta) - conclusión (evaluación)] sería propio de las secuencias denominadas "explicativas". Sin embargo, en el caso que planteamos no se reproduce la sucesión de actos de habla que se derivan de este esquema, puesto que la presentación de un hipotético "problema" mediante una pregunta se "autorresuelve" al ser la pregunta un acto de habla falaz que esconde una aserción indirecta y no la petición de resolución de una incógnita. 
saber que eres gay. ¿En qué cambia una persona cuando decide aceptar su condición de homosexual para que cambie tanto el trato de los amigos o conocidos? No cambiamos, simplemente somos sinceros con nosotros mismos. Dejamos de vivir la vida de los demás para vivir la nuestra propia. Dejamos de escondernos, de mentir, y empezamos a ser nosotros. La única faceta que cambia es reconocer nuestra opción sexual.

Nunca podré entender que una persona que dice ser liberal llegue a decir que tienes que pedirle perdón sólo por haberle dado un beso a un chico delante suyo. Si ese beso se lo hubiese dado a una chica, ¿también tendría que pedirle perdón? Espero que llegue el día que este tipo de cosas no pasen, que se acabe con la intolerancia y nos dejen de mirar como bichos raros para vernos como lo que somos: personas normales.

Francisco Luis Moya [La Vanguardia, 25/09/2003]

En (6) y en la segunda IR de (7) el cometido de ambos enunciados interrogativos es aportar la causa que justifica, en (6), el enunciado previo, es decir, el hecho de haber prescindido de la ONU para declarar la guerra; y en (7) una polaridad determinada (la negativa) para la apódosis de la estructura condicional. En las dos muestras el emisor no plantea una verdadera pregunta. Así, en (6) la IR transmite la aserción indirecta no la necesitaban para nada que, en el marco del texto, se analiza como la causa del evento descrito anteriormente. En este caso, resultaría inaceptable intercambiar la IR por una hipotética respuesta no negativa en el caso de que tal enunciado se valorara como pregunta. En (7), paralelamente, toda la secuencia condicional pretende generar un eventual estado de cosas que se valore como el contraste a la situación antes señalada. A partir de aquí, pues, solo la inferencia no tendría que pedirle perdón permite asentar un devenir discursivo lógico, puesto que de lo contrario no existiría contraargumentación entre los dos eventos planteados. Así pues, la IR adquiere validez en ambos ejemplos precisamente por no disponer de un significado contrafactual como sucede en aquellos usos en los que la interrogación articula una pregunta, sino por completar relaciones oracionales truncadas formalmente por la modalidad interrogativa.

Si retomamos, a continuación, la explicación en torno al uso retórico del binomio pregunta-respuesta, podremos apreciar que una circunstancial emisión independiente o autónoma del primer enunciado interrogativo de (7) sin la correspondiente respuesta comportaría, probablemente como consecuencia de un topos, la lectura retórica de dicho enunciado. En este sentido, pues, queda fuera de lugar argumentar que la retoricidad como valor pragmático aparece relacionado con una información catafórica. El emisor utiliza el esquema dual de la modalidad interrogativa, es decir, aprovecha que en su vertiente extensiva el formato interrogativo, precisamente por articularse como una estructura abierta o inconclusa, permite añadir un segmento de cierre discursivo. Sin embargo, la presencia de un material lingüístico destinado a cerrar la estructura prototípica interrogativa no responde en esta ocasión a resolver la cuestión que, en primer término, se ha planteado como incógnita. El uso retórico del esquema interrogativo tiene una doble explicación: por un lado, sirve como estrategia de refuerzo argumentativo, en clara convergencia con los marcadores aditivos, ya que se incide doblemente sobre la misma idea (¿En qué cambia una persona...? No cambiamos... equivale a afirmar Una persona no cambia en nada cuando decide aceptar su condición... No cambiamos). Por otro, actúa como un marcador reformulativo, ya que retoma el contenido previo para replantearlo con un formato distinto. De nuevo, pues, la posibilidad de análisis paralelo entre marcadores e IR se hace evidente. 
En (8), ya para finalizar, se presenta una CAD en la que se pueden apreciar de forma combinada algunos de los valores textuales que hemos ido describiendo en torno a la IR. Hallamos, en (8a), una IR con función de apertura textual que, de nuevo, actúa no solo como estructura dinamizadora del discurso, sino también como premisa sobre la que se asienta un argumento por analogía, que se refuerza con (8c) y (8d), que a su vez sirven como paralelismos para las situaciones descritas en sus respectivos comentarios posteriores. Asimismo, la retoricidad en (8a, c y d) supone, desde una perspectiva textual, el conjunto de causas que sustentan el valor retórico de (8b). Finalmente, (8e) presenta la función prototípica de cierre que, en esta ocasión, actúa a modo de conclusión-resumen de todo el proceso argumentativo.

(8) Deudas como losas

(a) ¿Estaríamos dispuestos a cobrarle a un vecino una deuda aun sabiendo que necesita esos recursos para alimentar a sus hijos? Estos días se está discutiendo en el Parlamento una propuesta de ley reguladora del tratamiento de la deuda externa que da la oportunidad de aliviar una de las principales losas que ahogan la vida de miles de personas en los países del sur.

Muchos de los países deudores destinan anualmente un importe igual a su presupuesto en educación o sanidad a sufragar esa deuda. (b)¿Tenemos derecho a imponer unos pagos que impliquen la muerte de miles de personas por no disponer de ninguna asistencia sanitaria? (c) ¿Estariamos dispuestos a pagar una deuda que contrajo el presidente de nuestra escalera y que gastó comprando un anillo de oro para su mujer? Pues muchas de las deudas que los países del sur están pagando fueron contraídas por gobiernos corruptos y los préstamos fueron colocados directamente en paraísos fiscales. (d) ¿Estaríamos dispuestos a pagar una deuda que contrajo el administrador de fincas cuando contrató a unos matones para amenazarnos y asegurarse así cuatro años más como gestor del edificio? En muchos casos las deudas fueron contraídas por dictadores que las usaron para comprar armas y reprimir a la población.

(e) ¿Es lógico que sean los nietos de los que recibieron las balas los que ahora estén pagando esa deuda?

Marc Porté [La Vanguardia, 02/03/2006]

\section{Conclusiones}

En este artículo se ha propuesto un análisis textual para un fenómeno de índole pragmática como es la IR. El objetivo último ha sido demostrar que el valor pragmático de retoricidad no es ni casual ni gratuito en la cohesión y coherencia de un texto porque, de hecho, (i) contribuye a ordenar el material lingüístico e informativo del texto estableciendo unas estructuras más o menos estables, (ii) repercute en la composición estructural de los esquemas argumentativos prototípicos matizando, en ocasiones, la fuerza y orientación argumentativa del contenido proposicional que incorpora y (iii) permite marcar unas imbricaciones entre el contenido discursivo y la actitud del emisor frente a este. La comparación asentada en relación con los parámetros descriptivos marcados por Briz (1998) en torno a los marcadores del discurso buscaba, salvando todas las diferencias, sintetizar unas tendencias de uso en torno a la IR que permitieran analizar el potencial valor cohesivo de esta estrategia pragmática. Asimismo, el hecho de abordar el estudio de la IR desde una vertiente textual sustentada en muestras reales confirma la necesidad de subordinar el estudio de esta estrategia pragmática a perspectivas que partan de un análisis discursivo, puesto que el significado indirecto que incorpora la IR 
se explica como consecuencia de las relaciones de significado que se deducen del texto. Quedan, en todo caso, estudios pendientes que aborden, por ejemplo, hasta qué punto el género discursivo determina estas funciones y usos, $\mathrm{u}$ otros que se centren en aspectos no tratados con profundidad en este trabajo como las secuencias compuestas por estructuras interrogativas múltiples o el valor directivo derivado de un doble proceso de interpretación inferencial en torno a la IR.

\section{Referencias bibliográficas}

Adam, J. M. (1990): Éléments de linguistique textuelle. Bruselas-Lieja, Mardaga.

Adam, J. M. (1992): Les textes: types et prototypes. París, Nathan.

Adam, J. M. (2005): La linguistique textuelle. Introduction à l'analyse textuelle des discours, París, Armand Colin.

Anzilotti, G. I. (1982): «The rhetorical question as an indirect speech devide in english and Italian», Canadian Modern Language Review, 38, págs. 290-302.

Beaugrande, R. y W. U. Dressler (1981): Introduction to Text Linguistics. Londres-Nueva York, Longman.

Bernárdez, E. (1982): Introducción a la lingüística del texto. Madrid, Espasa-Calpe.

Bernárdez, E. (1995): Teoría y epistemología del texto. Madrid, Cátedra.

Borillo, A. (1981): «Quelques aspects de la question rhétorique en Français», DRLAV, 25, págs. 1-33.

Briz, A. (1998): El español coloquial en la conversación. Esbozo de pragmagramática. Barcelona, Ariel.

Brown, G. y G. Yule (1983): Análisis del discurso. Madrid, Visor.

Calsamiglia, H. y A. Tusón (1999): Las cosas del decir. Barcelona, Ariel.

Carranza, I. (2001): «Argumentar, explicar y justificar con preguntas retóricas», Revista Iberoamericana de Discurso y Sociedad, 3-2, págs. 61-83.

Casado Velarde, M. (1993): Introducción a la gramática del texto en español. Madrid, Arco Libros.

Escandell Vidal, M. V. (1984): «La interrogación retórica», Dicenda, 3, págs. 9-38.

Escandell Vidal, M. V. (1988): La interrogación en español: semántica y pragmática. Madrid, Ediciones de la Universidad Complutense.

Estébanez Calderón, D. (1998): Diccionario de términos literarios. Madrid, Alianza Editorial.

Fernández García, F. (2002): «Sobre la funcionalidad de la interrogación retórica en la oratoria políticoelectoral». En Actas del IV Congreso de Lingüistica General. Cádiz, Servicio de publicaciones de la Universidad de Cádiz, págs. 989-998.

Frank, J. (1990): «You call that a rhetorical question? Forms and functions of rhetorical question in conversation», Journal of Pragmatics, 14, págs. 723-738.

González Calvo, J. M. (1996): «Semántica y sintaxis. El caso de las interrogaciones retóricas». En Rejas Rodríguez, M ${ }^{\mathrm{a}}$ T. y G. Salvador (eds.): Philologica. Homenaje al profesor Ricardo Senabre. Cáceres, EUEX, págs. 225-240.

Halliday, M. A. K. (1994): An introduction to functional grammar. Londres, Edward Arnold.

Halliday, M. A. K. y R. HASAN (1976): Cohesion in English. Londres, Longman.

Han, C. (2002): «Interpreting interrogatives as rhetorical questions», Lingua, 112, 3, págs. 201-230.

Haverkate, H. (1994): «Forma y estilo de la interacción verbal en La Celestina: la retoricidad de la pregunta retórica», Foro Hispánico, 8, págs. 41-54.

Igualada Belchí, D. A. (1994): «Estrategias comunicativas. La pregunta retórica en español», Revista Española de Lingüística, 24, 2, págs. 329-344. 
Ilie, C. (1994): What else can I tell you? A pragmatic study of english rhetorical questions as discursive and argumentative acts. Estocolmo, Almqvist \& Wiksell International.

Koshik, I. (2005): Beyond rhetorical questions. Assertive questions in everyday interaction. Ámsterdam, John Benjamins.

Mederos, H. (1988): Procedimientos de cohesión en español actual. Tenerife, Publicaciones del Cabildo Insular de Tenerife.

Rohde, H. (2006): «Rhetorical questions as redundant interrogatives», San Diego Linguistics Papers, 2, págs. 134-168.

Schmidt-Radefeldt, J. (1977): «On so-called rhetorical question», Journal of Pragmatics, 1-1, págs. 315-345.

Van Dijk, T. A. (1980): Texto y contexto. Madrid, Cátedra.

Van Dijk, T. A. (1983): La ciencia del texto. Barcelona, Paidós.

Werlich, E. A. (1976): Text Grammar of English. Heidelberg, Quelle \& Meyer.

Viehweger, D. (1990): «Savoir illocutoire et interprétation des texte», en Charolles, M. et al. (eds.), Le discours. Représentations et interpétations. Nancy, Presses universitaries de Nancy, págs. 41-51.

\title{
Obras y fuentes utilizadas
}

\author{
$A B C$ (España) \\ El País (España) \\ La Vanguardia (España).
}

